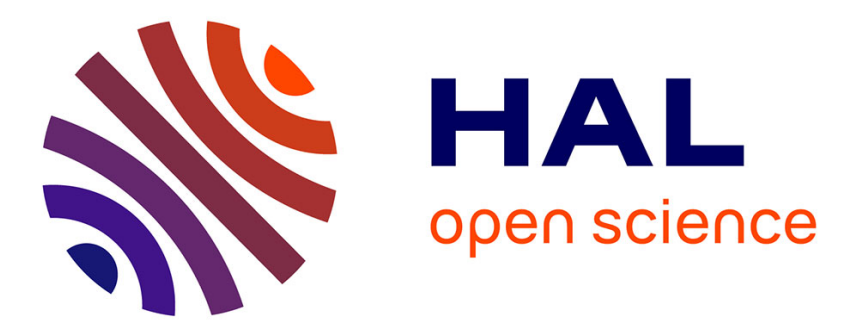

\title{
Unified Graphical Co-Modelling of Cyber-Physical Systems using AADL and Simulink/Stateflow
}

Haolan Zhan, Qianqian Lin, Shuling Wang, Jean-Pierre Talpin, Xiong Xu, Naijun Zhan

\section{- To cite this version:}

Haolan Zhan, Qianqian Lin, Shuling Wang, Jean-Pierre Talpin, Xiong Xu, et al.. Unified Graphical Co-Modelling of Cyber-Physical Systems using AADL and Simulink/Stateflow. UTP 2019 - 7th International Symposium on Unifying Theories of Programming, Oct 2019, Porto, Portugal. pp.1-20, 10.1007/978-3-030-31038-7_6 . hal-02193662

\section{HAL Id: hal-02193662 https://hal.inria.fr/hal-02193662}

Submitted on 24 Jul 2019

HAL is a multi-disciplinary open access archive for the deposit and dissemination of scientific research documents, whether they are published or not. The documents may come from teaching and research institutions in France or abroad, or from public or private research centers.
L'archive ouverte pluridisciplinaire HAL, est destinée au dépôt et à la diffusion de documents scientifiques de niveau recherche, publiés ou non, émanant des établissements d'enseignement et de recherche français ou étrangers, des laboratoires publics ou privés. 


\title{
Unified Graphical Co-Modelling of Cyber-Physical Systems using AADL and Simulink/Stateflow
}

\author{
Haolan Zhan ${ }^{1,2}$, Qianqian $\operatorname{Lin}^{1,2}$, Shuling Wang ${ }^{1}$, Jean-Pierre Talpin ${ }^{3 \star}$, \\ Xiong $\mathrm{Xu}^{1}$, and Naijun Zhan ${ }^{1,2(\bowtie)}$ \\ 1 State Key Lab. of Computer Science, Institute of Software, CAS, Beijing, China \\ ${ }^{2}$ University of Chinese Academy of Sciences, Beijing, China \\ 3 Institut National de Recherche en Informatique et en Automatique (INRIA) Rennes, France \\ \{zhanhl, linqq, wangsl, xux, znj\}@ios.ac.cn, \\ jean-pierre.talpineinria.fr
}

\begin{abstract}
The efficient design of safety-critical embedded systems involves, at least, the three modelling aspects common to all cyber-physical systems (CPSs): functionalities, physics and architectures. Existing modelling formalisms cannot provide strong support to take all of these three dimensions into account uniformly, e.g., AADL is a precise formalism for modelling architecture and prototyping hardware platforms, but it is weak for modelling physical and software behaviours and their interaction. By contrast, Simulink/Stateflow is strong for modelling physical and software behaviour and their interaction, but weak for modelling architecture and hardware platforms. To address this issue, we consider the combination of AADL and Simulink/Stateflow, two widely used graphical modelling formalisms for CPS design in industry. This combination provides a unified graphical co-modelling formalism supporting the design of CPSs from all three software, hardware and physics perspectives uniformly. This paper focuses on the required concepts to combine them together, and outlines how to verify and simulate a system model defined using the combined graphical views of its constituents, by considering the case study of an Isollete System.
\end{abstract}

Keywords: AADL · Simulink/Stateflow · Co-simulation · Code generation · Analysis

\section{Introduction}

Cyber-physical systems (CPSs), networked embedded systems (IoT, sensor networks), exploit computing units to monitor and control physical processes via wired or wireless communication. CPSs are omnipresent, from high-speed train control systems, power and control grids, automated plants and factories, transportations, to ground, sea, air and space. Most CPSs are entrusted with mission- and safety-critical tasks. Therefore, the efficient and verified development of safe and reliable embedded systems is a priority mandated by many standards, yet a notoriously difficult and challenging research domain.

$\star$ Jean-Pierre Talpin is partially supported by Nankai University 
As to standards, model-based design (MBD) has become a predominant development approach in the embedded system industry. In the MBD methodology, the development of a system starts by a model, based on which extensive analysis and verification are conducted, so that errors can be identified and corrected as early as possible, and at best before the system is implemented or built.

Subsequently, abstract system-level models are refined to semantically more concrete models and to source code, by model-transformation. The merits of MBD is hence to, at least, include the following folds:

- Complexity becomes tractable and controllable, thanks to system level abstraction.

- Errors can be identified and corrected at the very early stages of system design.

- Correctness and reliability can be guaranteed by proving the refinement process and all decomposed subsystems (components).

- Developers can fully reuse existing components and/or systems, to improve development efficiency even more.

Unsurprisingly, available formalisms and environment for CPS design are numerous, e.g., hybrid automata, Hybrid CSP (HCSP), differential logic, Ptolemy, Metropolis, C2E2, etc. in academia; Simulink/Stateflow, Modelica, SCADE, Labview, etc., in industry; UML, SysML, MARTE and so on, for MBD. Because of the tight coupling of hardware, software, and physics in CPS design, one has to model a complex CPS from the perspectives of functionality (software), physicality (physical environment and hardware platform), and architecture uniformly, but unfortunately, most of existing modelling techniques do not support all of these three aspects well and uniformly.

For instance, the Architectural Analysis \& Design Language (AADL) [12] is an architectural-centric model-based language developed by SAE International. It features strong capabilities to describe the architecture of a system due to the pragmatic (and practice-inspired) effectiveness of combining software and hardware component models. Meanwhile, it also supports the formal description of discrete behaviour using its BLESS Annex. Thanks to its succinct syntax, effective functionality and facilitated extensibility (by annexes i.e. plugins), AADL has been widely exploited in various embedded system domains, e.g., avionics, automotive. However, the core of the AADL only supports for modelling embedded system hardware structures and abstraction of its relevant discrete behaviour relevant to verification. It does not support the description of the continuous physical processes to be controlled by the embedded system and its combination with software, although some attempts have been done $[4,16]$.

By contrast, Simulink [1] is the de facto standard toolbox that has demonstrated strong capabilities for the model-based analysis and design of signal processing systems. It contains a large palette of functional blocks and supports their composition by continuous-time synchronous data-flow, as well as an intuitive graphical modelling language reminiscent of circuit diagrams. It is thus appealing to practitioners and engineers for whom it is designed for. Moreover, Stateflow [2] is a toolbox adding facilities for modelling and simulating reactive systems by means of hierarchical statecharts, extending Simulink's scope to event-driven and hybrid forms of embedded control.

However, Simulink/Stateflow can hardly model system architectures and hardware platforms. To address this issue, we complement Simulink with AADL to provide a 
unified graphical modelling formalism to support all the three perspectives of CPS design uniformly. The overview of the combination is given in Fig. 1. Our basic idea to

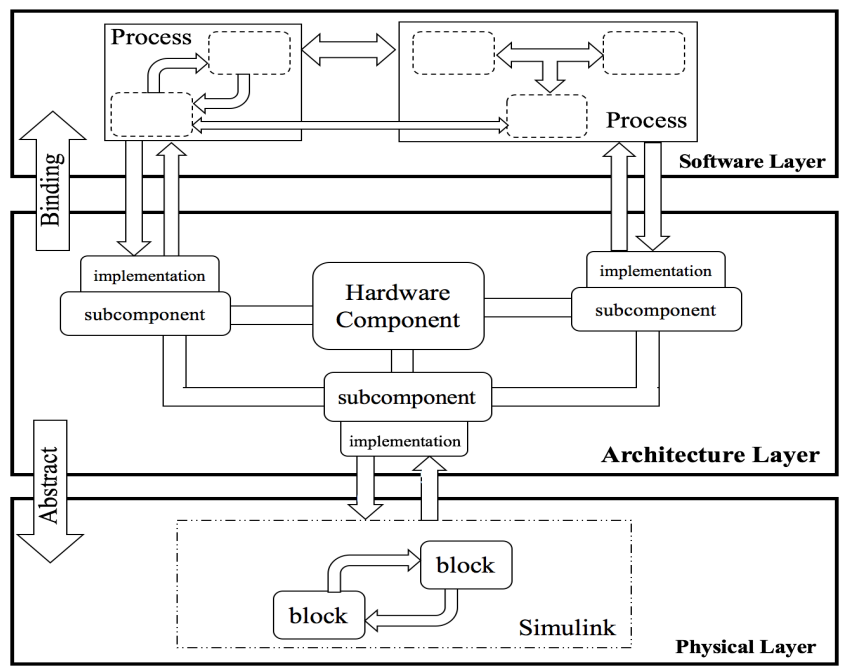

Fig. 1: An overview of the combination of AADL and Simulink

combine AADL and Simulink/Stateflow together can be sketched as follows:

System architecture and hardware platform: are given as AADL components.

Software behaviour: is modelled either as AADL components or Simulink/Stateflow diagrams.

Physical processes and its interaction with software: are modelled as Simulink/Stateflow diagrams.

Type classifier generation for Simulink/Stateflow diagrams: In order to put all AADL components and Simulink/Stateflow diagrams together, and build a view of the whole system by computing type classifiers for each Simulink/Stateflow diagram.

First, we translate Simulink/Stateflow diagrams into HCSP to obtain a formalisation of their port declarations [34,29,33]. Second, we use Daikon [11], or invariant generation [19] or, possibly, compositional proof tactics [21] to associate type classifiers with formal contracts.

Simulation of the whole graphical model, defined by the combination of AADL and Simulink views, amounts to coordinating code generated by both AADL and Simulink model simulators through effective port communications. Verification of the combined models are performed by translation to HCSP [18,34,28,7].

Contribution. In this paper, we propose a unified graphical framework using AADL and Simulink to model, simulate and verify cyber-physical systems. This framework depicts a methodology to design and simulate CPSs in a unified graphical environment 
while supporting formal verification of its functional, physical and structural artifacts uniformly using HCSP. Our graphical framework consists of AADL, its BLESS Annex and Simulink/Stateflow. It is implemented by a simulation environment called AADLSim, which integrates a set of tools, including an automatic translator from AADL into C, and a simluation engine combining AADL and Simulink/Stateflow models. To demonstrate the above framework and tool, the case study of an Isolette System is provided.

Paper Organization. The rest of the paper is organized as follows. Sec. 2 provides an overview of AADL, Simulink/Stateflow, and the notion of design by contract. Sec. 3 presents the Isollete case study, which will be used as a running example throughout the paper. Sec. 4 depicts our combined framework composed of AADL and Simulink/Stateflow, especially how to compute the type classifiers and define the contracts for Simulink/Stateflow diagrams. Sec. 5 presents in detail how to implement the co-modelling and co-simulation in the unified framework. Sec. 6 gives the related work and Sec. 7 concludes this paper and discusses some future work.

\section{Preliminaries}

In this section, we first provide an overview of the AADL standard, by highlighting its structure and BLESS Annex, then introduce Simulink/Stateflow with most relevant features of it. Finally, we briefly introduce the notion of design by contract.

\subsection{AADL}

AADL provides means to specify both the application software and the execution hardware of an embedded system, and supports textual, graphical and XML Metadata Interchange (XMI) specification formats. Components with type and implementation classifiers are instantiated and connected together to structure the system architecture. The AADL core language constructs are categorised into application software, execution platform and composite components. A system component represents a composite entity containing software, execution platform and system components.

Components and Connections The execution platform category represents computation and communication resources including processor, memory, bus and device components. A processor component represents the hardware and software responsible for scheduling and executing task threads. Properties can be assigned to a processor component to specify scheduling policies, high-level operating system services and communication protocols. A memory component is used to represent storage entities for data and code. A device component can model a physical entity in the external environment: a plant or the software simulation of the plant. It can also be used as an interactive component like sensor or actuator. A bus component represents the physical connections among execution platform components.

Application software category consists of process, data, subprogram, thread, and thread group components. A process component represents the protected address space, 
which is bound to a memory component. A data component can be used to abstract data type, local data or parameter of a subprogram. A subprogram models the executable code which is called, with parameters, by thread and other subprograms. Thread is the only schedulable component with execution semantics to model system execution behavior. A thread represents sequential flow of the execution and the associated semantic automation describes life cycle of the thread.

A component type declaration defines interface elements and may contain features. Features comprise data, event and event data ports to transmit and receive data, control, and data/control respectively. Port communication is typed and directional. An in port receives data/control and an out port sends data/control while an in out port can send and receive data/control. Communication is realized through connections between ports, parameters and access to shared data.

BLESS Annex. The Behavior Language for Embedded System with Software (BLESS) is a standardised annex independent of the core AADL language. BLESS extends AADL with the ability of specifying behaviour of component interfaces, providing formal semantics for AADL behavioural descriptions and automatically generating verification conditions to be proven. BLESS models state machines using guards and actions to give precise specifications of discrete hardware/software behaviours. BLESS also introduces assert and invariant sections in AADL to specify assertions and predicates that behavioural models must satisfy.

We refer to AADL standard document AS5506-B [26] for further details.

\subsection{Simulink/Stateflow}

Simulink is an environment for model-based design of dynamical systems, and has become a de facto standard in the embedded systems industry. It provides an extensive library of pre-defined blocks for building and managing block diagrams, and also a rich set of fixed-step and variable-step solvers for simulating dynamical systems. It also provides features such as subsystems for building large systems in a hierarchical way. Stateflow is a toolbox adding facilities for modelling and simulating reactive systems by means of hierarchical statecharts. It extends Simulink scope to event-driven and hybrid forms of embedded control.

A Simulink model contains a set of blocks, subsystems, and wires, where blocks and subsystems cooperate by dataflow through the connecting wires. An elementary block receives input signals and computes output signals according to user-defined parameters altering its functionality. One typical parameter is sample time, which defines how frequently the computation is performed. Blocks are classified into two types: continuous blocks with sample time 0 , and discrete blocks with positive sample time. For continuous blocks, the continuous state changes over time continuously, e.g. the position or the speed of a moving car. It is usually represented by an ordinary differential equation (ODE). Simulink provides an amount of ODE solvers for solving ODEs based on the numerical integration methods.

Stateflow offers the modelling capabilities of statecharts for reactive systems. It can be defined as Simulink blocks, fed with Simulink inputs and producing Simulink outputs. A stateflow diagram is composed of transitions, states and junctions. Each transi- 
tion connects a source state to a destination state. It it is labelled with $E[C]\{c A c t\} / t A c t$, where $E$ is an event, $C$ is the condition, $c A c t$ the condition action, and $t A c t$ the transition action. The event $E$ triggers the transition to take place, provided that the condition $C$ is true. As soon as $C$ evaluates to true, the action $c A c t$ will be executed immediately, while $t A c t$ will be left pending and put in a queue first, and will be executed until a valid transition path is completed. A state is labelled by three optional types of actions: entry action, during action, and exit action.

Stateflow supports to construct flow charts using connective junctions and transitions, which can be used between states to specify decision logics to form transition networks. The Stateflow states can be composed to form hierarchical diagrams: Or diagram, for which the states are mutually exclusive and only one state becomes active at a time, and And diagram, for which the states are parallel and all of them become active simultaneously.

Being based on a large palette of individually simple function blocks and their composition by continuous-time synchronous dataflow as well as the modelling capabilities of statecharts for reactive systems, Simulink/Stateflow offers an intuitive graphical modelling language of CPSs for practicing engineers. Ordinary users can quickly build the model's framework by connecting the corresponding graphical modules and defining interfaces. Therefore, it is convenient and efficient to design and analyse the components using Simulink/Stateflow for co-simulation.

\subsection{Design by Contract}

Design by contract $(\mathrm{DbC})$ is an engineering methodology whereby system designers should define semantically founded, precise and verifiable interface specifications for hardware and software components. These specifications extend the ordinary notion of abstract data type with logical properties describing the pre-conditions, post-conditions and invariants of a software function or of a hardware block.

The term design-by-contract is due to Bertrand Meyer in connection with the definition of the Eiffel programming language and his book Object-Oriented Software Construction [22]. It is rooted in Hoare logic, where the contract $(A, G)$ of a program $P$ naturally corresponds to the provable assertion $C \vdash\{A\} P\{G\}$ in some logical context $C$. Contracts has been algebraically meta-theorized by Benveniste et al. [6], systematically applied to model-based design frameworks like BIP [5].

Recently, [21] extended the reach of design-by-contract to the case of modularly verifying hybrid system models by the introduction of contracts in a compositional design methodology for Differential dynamical Logic (ddL) [24]. In this context, and by contrast, the contract of a given model $\Gamma \vdash[\alpha] \phi$ consists the evolution domain $H$ of the specification $\alpha$, as assumption, and differential invariant $\phi$, as guarantee.

\section{Isollete System: A Running Example}

In this section, we introduce the Isolette System as a running example throughout the paper. Isolette is an infant incubator described by the Federal Aviation Administration (FAA) in the Requirement Engineering Management Handbook (REMH) [17]. This 
example is concise enough to demonstrate, and also abundant enough to contain both discrete control behaviour and continuous plants, as a classical hybrid system [4].

\subsection{Isollete System}

The isollete example has been widely used to explain detailed behaviour for AADLbased development and new annexes, such as the BLESS Annex and the Error Model Annex [10]. Fig. 3 depicts the AADL graphical model of the isollete system, including processor, bus, sensor, actuator, controller, and controlled process with internal threads.

The system is used to maintain the temperature of the isollete box, a physical environment, within a desired range that is beneficial to infant. To this end, the controller obtains the temperature inside the box through the sensor, then computes an appropriate command to control the temperature through the actuator to switch on (off) the heater combined with the isollete box.

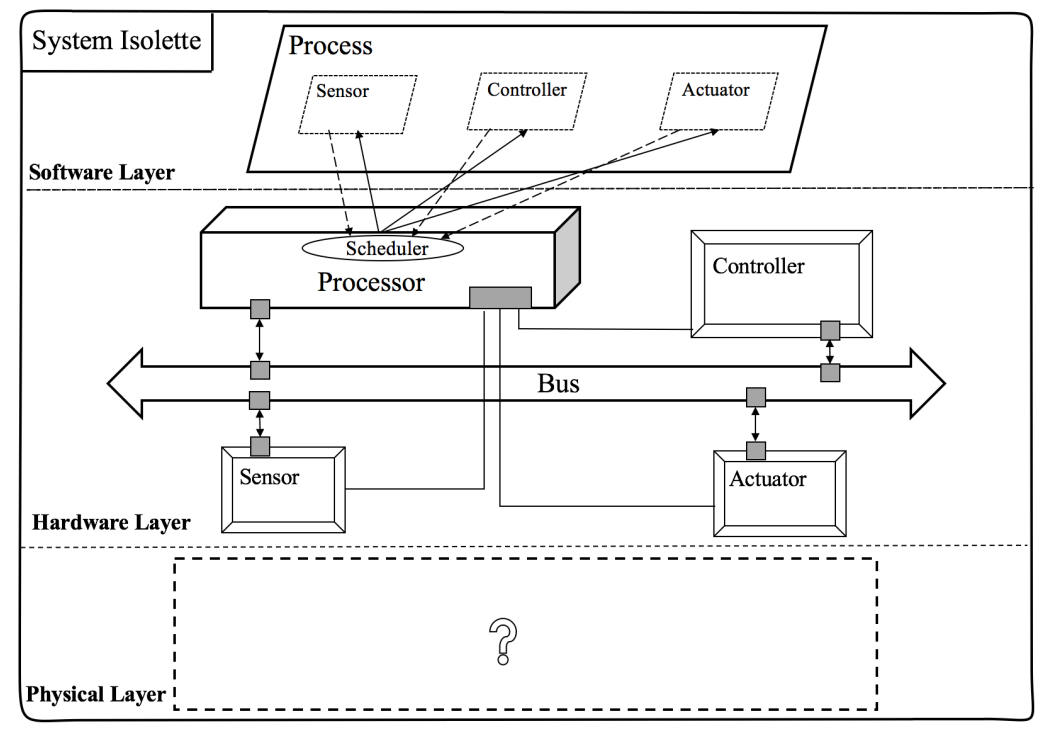

Fig. 2: AADL graphical model of Isolette system

The continuous evolution of temperature depends on the current status of the actuator. If the heater is on, the temperature will increase, otherwise decrease. According to the specification in the Section A.5.1.3 of the REMH [17], when the isolette is properly switched on, the temperature of the heater will change at a rate of no more than $1{ }^{\circ} \mathrm{F}$ per minute. Based on this specification, the temperature of the isollete box (denoted by $c$ ) and the temperature of the heater (denoted by $q$ ) are formally modelled by the ODEs (1) below. The constant 0.026 stands for the thermal conductivity. When the controller commands the actuator to switch the heater on, the rise in temperature $q$ will result in $c$ going up. In this specification, we assume that the room temperature outside the 
box to be constant at $73^{\circ} \mathrm{F}$, although its variations could also be modelled. The desired temperature inside the isollete box should be kept at a range between $97^{\circ} \mathrm{F}$ and $100^{\circ} \mathrm{F}$.

$$
\left\{\begin{array}{lll}
\dot{c} & =-0.026 \cdot(c-q) \\
\dot{q} & =1 & \text { if heater is on } \\
\dot{q} & =-1 & \text { if heater is off }
\end{array}\right.
$$

\subsection{Requirements}

Referring to environmental assumptions provided in the REMH, the following safety should be satisfied.

- Safety: The temperature inside the isollete box should be kept in between $97{ }^{\circ} \mathrm{F}$ and $100^{\circ} \mathrm{F}$, i.e., $97^{\circ} \mathrm{F} \leq c \leq 100^{\circ} \mathrm{F}$.

Moreover, considering the uncertainties from initial states, sensor errors, disturbance of dynamics, and numerical error caused by floating-point calculation, etc., it is required

- Stability and Robustness: The inside temperature $c$ will be finally steered towards the valid range after some time.

At this point, it is obviously hard to specify this physical model in using AADL and its annexes alone, notwithstanding its interaction with the digital controller, hence the question mark in Fig. 2 and the need for a complementary hybrid annex.

\section{Combination of AADL and Simulink/Stateflow}

The combination of AADL and Simulink/Stateflow aims at providing a unified graphical co-modelling formalism for CPSs, with which software, physical environment and execution hardware of a CPS can be modelled in a uniform framework.

\subsection{General Framework}

The Fig. 1 we already presented, describes the high-level architecture of the proposed unified graphical framework together with the connection among the three different physical, hardware and software layers. The architecture layer, described as AADL system composite components, specifies the types of hardware and software components, and (part of) their implementation (an abstraction of their actual implementation), as well as their composition. It usually consists of a central processor unit classifier with several subcomponent devices (like sensor, controller, and actuator etc.). Each of these classifiers has its own type and implementation. For software functionality and physical processes, the architecture layer usually needs their abstractions, i.e., the type classifiers of these software and physical components. The type classifier of a component declares the set of input and output ports, specifies the contract of its behaviour, that are accessible from outside. By contrast, the implementation classifier of a component binds its type classifier with a concrete implementation in the software and physical layers. 
Our framework provides two methods to describe the type classifier of a given Simulink/Stateflow model. The first one is to derive a type classifier, which is satisfied by the Simulink/Stateflow diagram, directly from its behaviour, see Sec. 4.2; the other is to define a contract in the style of an assume/guarantee pair, and then prove the given Simulink/Stateflow diagram to satisfy this contract, see Sec. 4.3.

In the software layer, software components are defined by their functionality, which can be done using either AADL or Simulink/Stateflow. In AADL, the functionality is defined by processes, and in each process, one or more threads may exist to describe specific controlling behaviours. The BLESS Annex can further be employed to specify the behavior of the system precisely. In order to establish stable communication between different processes, port declaration must be well defined. The AADL implementation in this layer will be binding to the corresponding software and hardware components in the architecture layer.

In the physical layer, the continuous behaviour of physical processes is implemented as Simulink/Stateflow diagram. In order to integrate the Simulink/Stateflow diagrams for implementing software or physical processes into the architectural layer, we need to define a type classifier for each Simulink/Stateflow diagram so that it can be assembled with other abstract components to form the architecture of the whole system at the architecture layer. We will explain the details of this process in the rest of this section.

Example 1. Now we can build a complete graphical model of the Isollete system with the combination as shown in Fig. 3, in which the Simulink/Stateflow diagram is given as Fig. 4.

Simulation. To simulate the graphical model with the combination of AADL and Simulink, we propose a cross-layer co-simulation framework, in which the hardware platform, control software, and physical dynamics in the designed CPS can be taken into account uniformly. We will explain the details of such specification in Sec. 5.

Verification. To further verify a graphical model given by the AADL-Simulink combination, we translate it into HCSP, which is an extension of CSP introducing differential equations to model the continuous evolution of the plant and three types of interrupts to model the interaction between continuous and discrete behaviours [13,31]. The formal verification of HCSP can be done along the lines of our previous work [18,32,28,7]. Moreover, the correctness of the translation can be strictly proved using higher-order UTP [8], which extends the classic Unifying Theories of Programming (UTP) [14] to hybrid systems by introducing higher-order quantifications and differential relations. The technical details of this part will be reported in a subsequent paper.

\subsection{Computing Type Classifier for Simulink/Stateflow Diagram}

As we explained above, when combining Simulink/Stateflow with AADL, we need to provide an abstraction for each Simulink/Stateflow diagram, i.e., its type classifier, so that it can be assembled with other components to form the whole system at the architecture layer, while the diagram itself will be used as the implementation classifier 


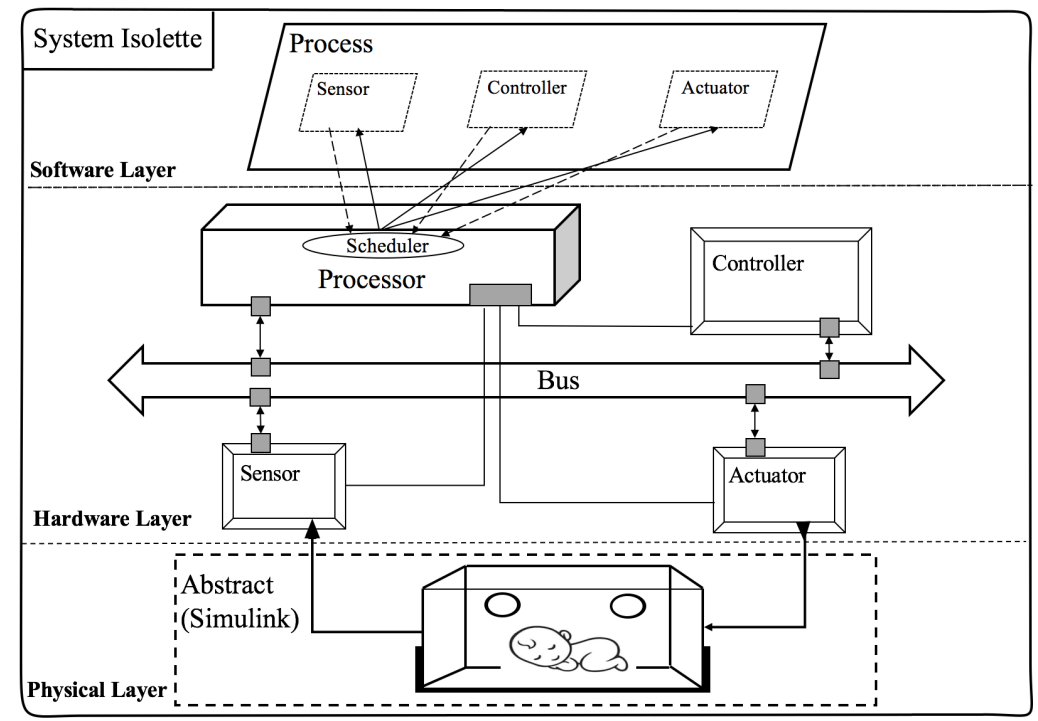

Fig. 3: AADL graphical model of Isolette system

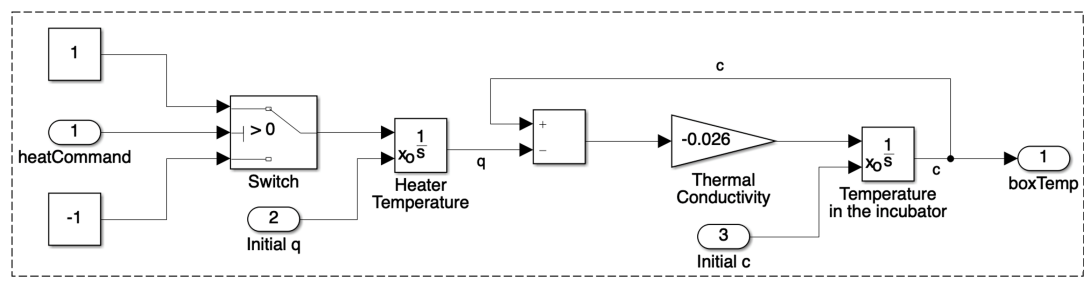

Fig. 4: Simulink model of Isollete box

of the component. Normally, the type classifier of a component consists of two parts: port declaration and constraints.

The port declaration declares a set of ports used to input and output data between the component and other ones. However, Simulink diagrams can be hierarchical, and hence its external ports can sometimes not be extracted directly. For example, consider the triggered subsystems in a Simulink diagram, they do not have any input and output ports, but are triggered by events. Therefore, we need to analyse the whole system in detail in order to obtain all external ports, particular, event ports. Moreover, this often gets worse when Stateflow models are additionally considered.

To address this problem, we exploit the tool Sim2 HCSP, a component in our toolkit MARS [7], which can translate a Simulink/Stateflow diagram into a formal HCSP process. By applying Sim2 HCSP, all external ports of a Simulink/Stateflow diagram can now be translated, and exposed, by a set of channels in the corresponding HCSP model, which is stored in a separate file. In the case of the Simulink diagram in Fig. 4, we can for instance obtain the following port declaration: 


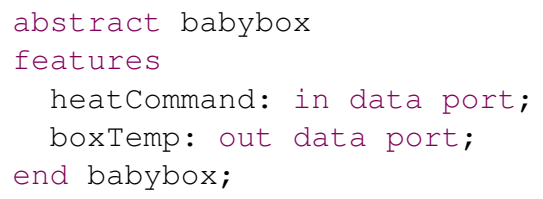

The reminder of the specification defines the contract of the component. It specifies the properties that should be satisfied by any execution of the component. In this paper, we adopt two approaches to generate the constraints for a given Simulink/Stateflow diagram. The first one uses Daikon [11]. The basic idea is to simulate the given Simulink/Stateflow diagram, and then run Daikon to generate a candidate invariant which satisfies all simulation runs. The more simulations are performed the more refined the generated invariant becomes. For example, considering the Simulink diagram in Fig. 4, by applying Daikon, we can obtain the following type classifier:

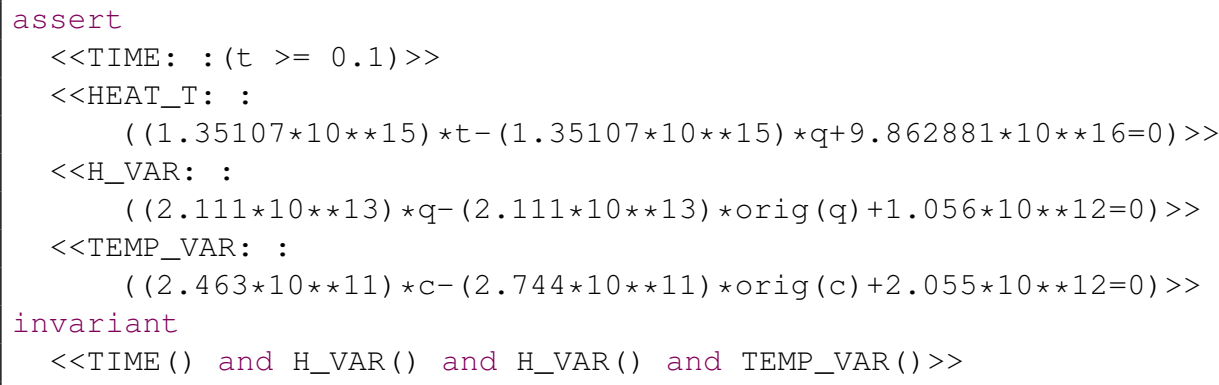

Alternatively, we can generate invariants directly from the Simulink/Stateflow diagram, or the translated HCSP process, by using technique of invariant generation for hybrid systems, e.g., [19]. Reconsider the Simulink diagram in Fig. 4, by using invariant generation, we can instead obtain the following type classifier:

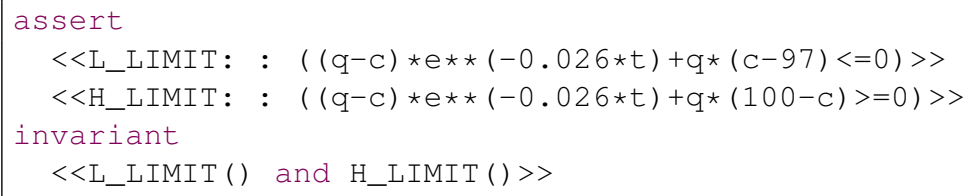

The efficiency of the first approach is much higher, but the generated invariant (approximation) can only be linear. Moreover, it may not become an actual invariant, even by conducting enough runs to refine it. By contrast, the second approach can generate more expressive and semantically correct invariants, but the efficiency is normally very low. How to improve the efficiency of invariant generation for hybrid systems is still a challenging problem.

\subsection{Defining Type Classifier as Contracts}

Our goal is to exploit the HCSP model provided by Sim2 HCSP [7] to support modular, component-wise analysis and verification of system models combined from architectures described in AADL and hybrid systems in Simulink/Stateflow. 
In the logical framework of HCSP, our model of contracts will naturally follow along the lines proposed by Lunel et al. for differential dynamic logic $[21,20]$. In that context, the contract of a specification $\alpha$ is defined by a pair $(A, G)$ of properties. $A$, the assumption, is a formula defining the evolution domain of $\alpha$ and $G$, the guarantee, is a formula stating its differential invariant.

In the Hybrid Hoare Logic for HCSP [18,27], these concepts are provided by the domain $B$ of derivatives $\dot{s}=e \& B$ and by pre- and postconditions, and history formulas $H F$, respectively.

It is hence tempting to adapt the composition theorem of [21] to the HCSP framework, as it provides a way to automate the proof of a system's contract, e.g. $\left(A_{1} \wedge\right.$ $A_{2}, G_{1} \wedge G_{2}$ ) from the (possibly tedious) proofs that its components $C_{i=1,2}$ satisfy the differential invariant $G_{i}$ in the domain $A_{i}$.

This theorem is obtained by obeying to the following associative-commutative definition of parallel composition of hybrid components [21, Def. 7]:

$$
\alpha \otimes \beta \widehat{=}\left(\operatorname{disc}_{\alpha} \cup \operatorname{disc}_{\beta} \cup\left(\operatorname{cont}_{\alpha} \oplus \operatorname{cont}_{\beta}\right)\right)^{*}
$$

which amounts to composing discrete components disc by using repetition and choice and continuous components cont by the union, noted $\oplus$, of there derivative equations. [21, Th. 2] proves the following: assume proof trees $\Gamma_{i} \vdash\left[C_{i}\right] G_{i}$ stating that the $G_{i}$ s are invariants of the components $C_{i}$ s in contexts $\Gamma_{i}$, for all $i=1,2$. Next, assume that the definitions in the $C_{i}$ s do not interfere, nor with the guarantees of all other $G_{j} \mathrm{~s}(i \neq j)$ :

$$
\forall i \forall j \neq i, \mathbf{d v}\left(C_{i}\right) \cap \mathbf{d} \mathbf{v}\left(C_{j}\right)=\emptyset \wedge \mathbf{d} \mathbf{v}\left(C_{i}\right) \cap \mathbf{u v}\left(G_{j}\right)=\emptyset
$$

with $\operatorname{dv}(C)$ denoting the variables defined by a component and $\mathbf{u v}(G)$ the variables used or referenced in a guarantee $G$. Then, there always exists a derivation of $\Gamma_{i=1,2} \vdash$ $\left[\otimes_{i=1,2} C_{i}\right]\left(\wedge_{i=1,2} G_{i}\right)$, which the proof of the theorem mechanically constructs: an automated proof tactic.

This model of compositional contracts can be employed to implement Sangiovanni's "meet in the middle" design methodology as the mitigation of software, hardware and physics constraints from the viewpoint of the target system architecture. In the case of the isolette, for instance, it can be used to verify the safety requirement of the isollette in nominal mode, Sec. 3.2 (i.e. after an initialization period) by cross-validating the differential invariant of the physical model with the (adequate) operations of its controller on the sensors and actuators, all four expressed by separate logical contracts. A use case of this method with KeymaeraX, concerning the well-known controlled watertank problem, is given in Lunel's $\mathrm{PhD}$ Thesis [20].

\section{Co-Modelling and Co-Simulation}

This section details the implementation of the unified framework introduced in Subsec. 4.1 for designing and analyzing CPSs. The design flow of the framework is shown in Fig. 5. It consists of three stages: co-modelling, model translation, and co-simulation.

In the co-modelling stage, designers can exploit the toolkit OSATE/AADL and Matlab/Simulink to model different parts of systems. Port definitions are required in each of 


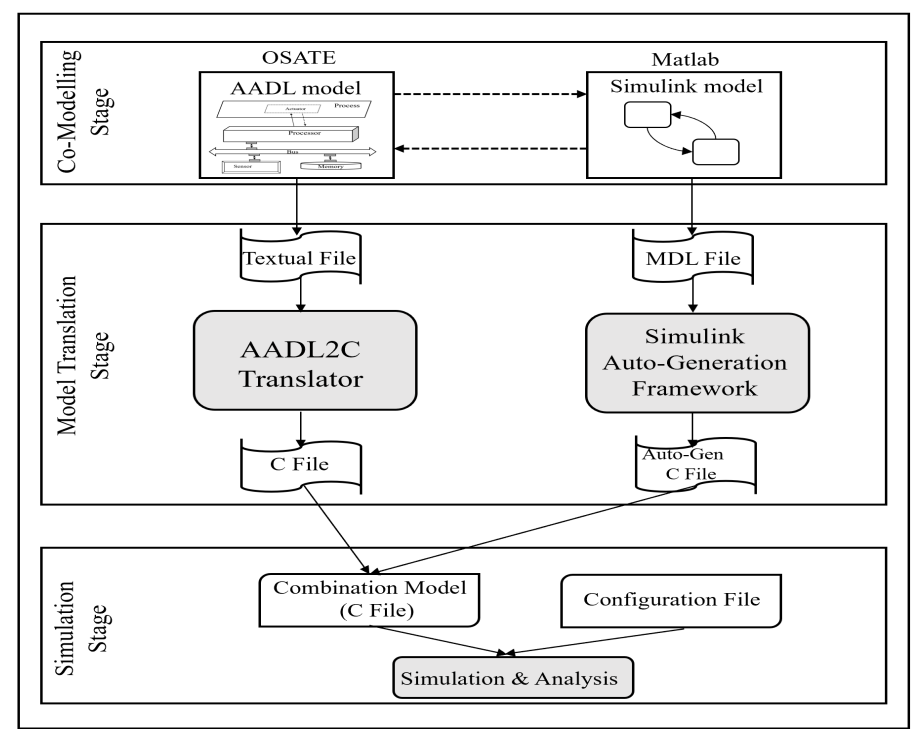

Fig. 5: Co-modelling and co-simulation of AADL and Simulink/Stateflow

the separate parts in order to establish the connection between AADL and Simulink/Stateflow models. Then, in the model translation stage, in order to integrate the two separate models and analyse them as a whole, we translate both the AADL and Simulink/Stateflow models into $\mathrm{C}$ code. On one hand, we developed a toolkit named AADL2C Translator, a novel code generation plugin to parse the AADL standard textual file and transfer it into $\mathrm{C}$ code. On the other hand, auto generation of $\mathrm{C}$ code from Matlab/Simulink models is done directly by using the Real Time Workshop(RTW) toolbox of Matlab. In the co-simulation stage, the translated C code from AADL and Simulink will be combined at first by performing integration and parameter configuration. After that, the testbench files are generated for the model code. The model code together with the testbench files will be compiled by a $\mathrm{C}$ compiler, with the co-simulation results produced. The co-simulation results provide a feedback for engineers to analyse and revise their original designs in the modelling stage. All three stages are introduced in detail in the subsequent subsections.

\subsection{Co-Modelling in AADL and Simulink/Stateflow}

AADL modelling The OSATE platform provides two different design patterns for engineers to build AADL models: graphical models and textual code. To exploit the internal mechanism of AADL, we choose the textual form to build our system. With the BLESS Annex, AADL is also able to specify the discrete behaviour of components. AADL takes a top-down pattern to build a system: a system classifier is defined at the beginning, and then all its hardware and software subcomponents are declared in system implementation. For the Isollete example, the type classifier and implementation for the whole system shown in Fig . 3 are given as follows: 


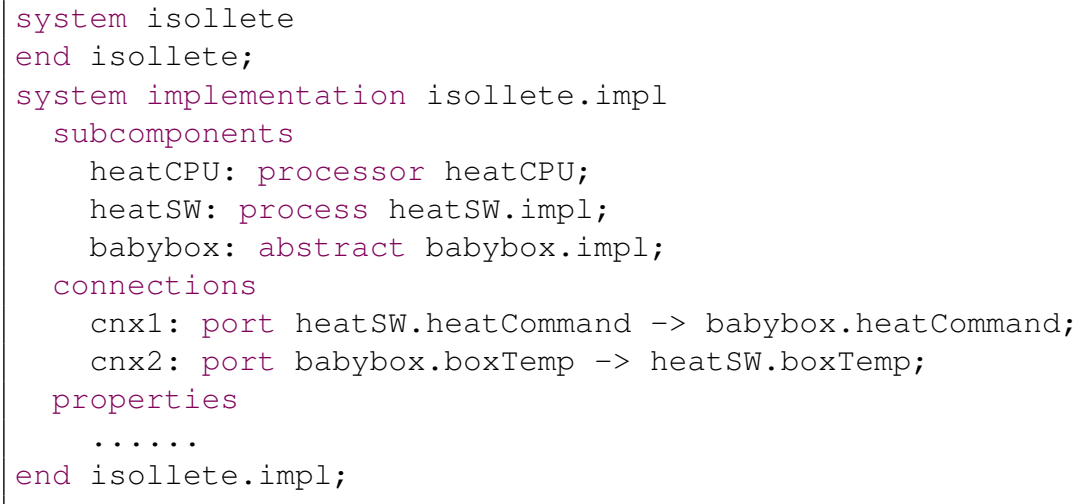

The heatCPU element defines the central processor. The heat $S W$ the central process for specifying the functionality of the sensor, the actuator and the controller. The babybox stands for the isollete box. In the connections section, the ports of heat $S W$ and the ports of babybox are connected, for transferring the heat command (representing the off or on status of the controlled variable) and the box temperature respectively. The properties section stipulates a binding relationship between the software and hardware subcomponents. We can omit the details of it here. The behaviours of the sensor, actuator and the controller are implemented as threads in AADL. In particular, the model of the controller is defined as follows:

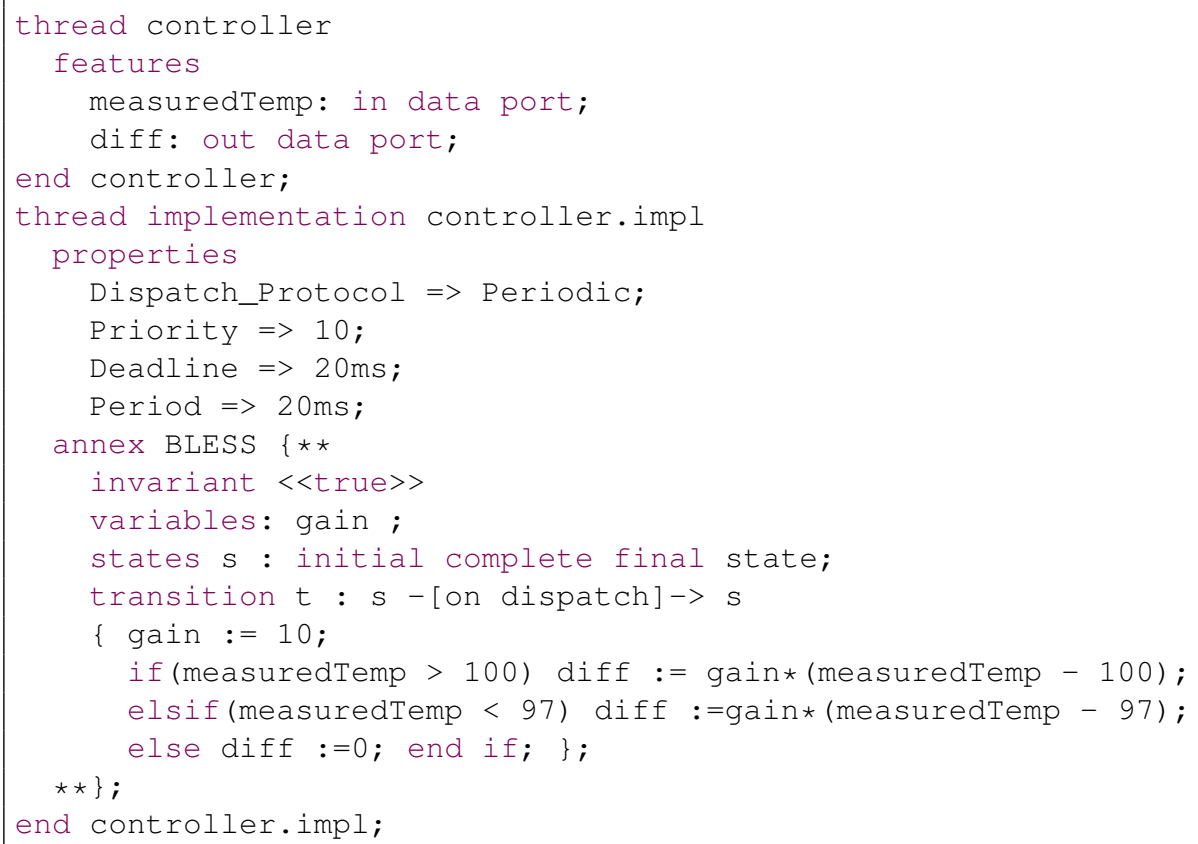


In the implementation, the controller defines the functionality using the BLESS Annex: the controller receives the measured temperature of the isollete box from the sensor via the input port measuredTemp, computes a difference between the temperature and the threshold, and then sends it to the actuator via output port diff. The actuator will decide whether to turn on or turn off the heat depending on that value.

Simulink/Stateflow modelling We will use Simulink/Stateflow to model the continuous behaviour of the CPS under design. For the Isollete box, we need to model the continuous behaviour defined by the ODE (1). The Simulink diagram has been given in Fig. 4.

Combination of models After building the models separately in AADL and Simulink/Stateflow, we will combine them to form the whole system. Our approach is to define abstract components in AADL and connect each of them to the corresponding Simulink/Stateflow models. For each abstract component, the type classifier declares all the ports connecting AADL and Simulink/Stateflow models, and the constraints for the actual behaviour. The abstract AADL type classifier of the isollete box is given in Sec. 4.2.

\subsection{Model Translation to C}

Translating the AADL model The translation from AADL to $\mathrm{C}$ is the most crucial part in the unified framework. It uses a collection of mapping rules from AADL concepts to $\mathrm{C}$ implemented by the compiler $A A D L 2 C$ Translator. Fig. 6 illustrates the model translation flow from AADL to C. AADL models can be defined in graphical or textual format: graphical models only describe the high-level architecture, while textual model includes the details such as the functional behaviours.

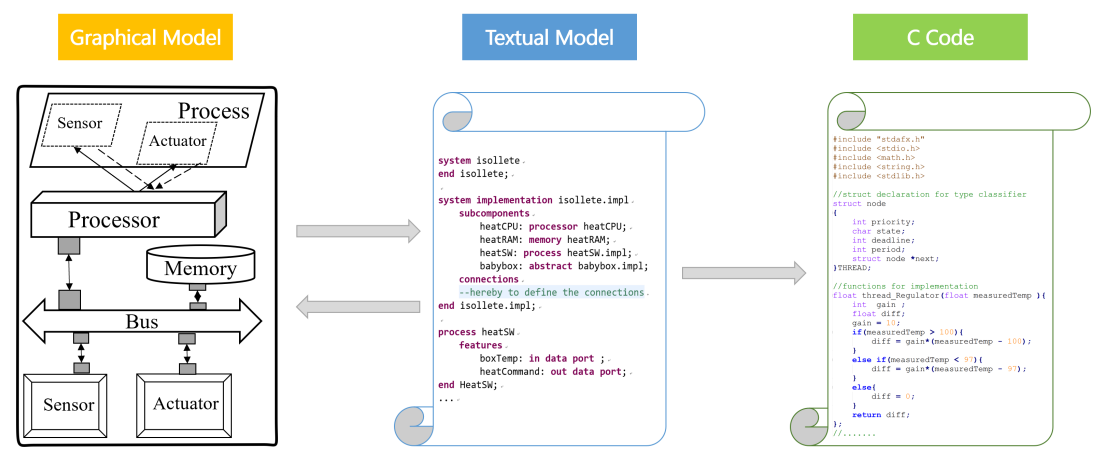

Fig. 6: An example illustrating the model translation flow from AADL to C

The compiler $A A D L 2 C$ Translator receives a source file as input and automatically generates the corresponding $\mathrm{C}$ code. According to the AADL grammar, a model is usually composed of several components, each with two parts: type declaration and 
implementation. The AADL2C Translator creates a struct object for each type declaration, e.g.. system, process, thread, etc., and defines a collection of properties of the corresponding type classifiers. The implementation classifier is translated into individual sub-functions, associated with relevant type classifiers and specific names. Especially, for thread implementation, two extra functions are specified for thread scheduling: create_thread() and thread_scheduling(). The create_thread() function adds a thread to a thread queue for dispatch, while the thread_scheduling() is designed to execute threads according to the scheduling protocol specified in the AADL model, e.g., RateMonotonic Scheduling (RMS), Highest Priority First (HPF), etc.

In order to implement the port communications between different components efficiently, an additional Global Port Data Management (GPDM) unit is introduced in the target $\mathrm{C}$ code. The GPDM will store all of the output ports as global variables, with names of the format componentType_componentName_outputPortName. In each simulation cycle, the values of these variables will be updated once.

Translating Simulink/Stateflow model Matlab provides an automatic code generation tool that helps to translate Simulink/Stateflow models into C code. It greatly improves the quality and efficiency of development and simulation. Here we briefly introduce the steps for generating $\mathrm{C}$ code files from Simulink/Stateflow models.

Before the translation, we simulate the Simulink/Stateflow models to be translated independently in Matlab, to guarantee that the models are executable. Then we use the Code Generation Tool provided in Matlab to generate $\mathrm{C}$ code. The model configuration process is carried out to set the Configuration Parameters, including the code generation method, the format of the generated model, etc. In detail, we need to configure the following three parts:

- Solver setting for the model solver. We set the solver's step size to be fixed length as RTW can only generate code from a fixed step model. Fixed-point solver provides a variety of algorithms including continuous methods and discrete methods. For the Isollete case study, we set the time step to be $0.1 \mathrm{~s}$.

- Hardware implementation provisions. The hardware implementation option is to specify the target hardware specification including the manufacturer and type of the chip, the word length, byte order of the chip and so on. In our framework, we set them as default.

- System object file of the model. The generated object files include a set of C source and header files. Suppose we call the Simulink/Stateflow model as box, the generated $\mathrm{C}$ files will include box.c, box_data.c, box.h, box_private.h and box_types.h. The box.h and box.c define the main behaviour of the original model, while box_data.c stores relevant constants or initial data, and the rest are the clarification header files.

Co-simulation requests a starting point of program execution so that we specify a main method as an interface to connect the C code generated from Simulink/Stateflow model with the $\mathrm{C}$ code interface generated from AADL model. 


\subsection{Co-simulation}

Now all the different parts of the system, including hardware components, application softwares and physical processes modelled in AADL and Simulink/Stateflow, have been translated into $\mathrm{C}$ code separately. In the co-simulation stage, we need to integrated all the separate $\mathrm{C}$ code files by defining the communication between them. The communication of distributed parts is implemented through the GPDM block mentioned above, which can be regarded as a global data memory storing all the data interfaces (external in/out ports) information of each component, such as AADL thread components and Simulink/Stateflow models. Local variables inside components are not considered by the GPDM block.

After the $\mathrm{C}$ code files are integrated, the simulation of the whole system can be performed. During simulation, it is allowed to set some configuration parameters, such as the global simulation clocks and the periodical simulation clocks. Besides, engineers can also assign different values to system variables in different simulation situations.

Simulation results of Isollete For the Isollete case study, we will check whether it fulfils the requirements mentioned in Sec. 3.2 by simulation. We first translate the AADL and Simulink model of the Isollete to $\mathrm{C}$ code, then consider two cases by setting different initial values for the variables. In the first case, both the temperature inside isollete box and heat actuator are initially set as $73^{\circ} \mathrm{F}$, same as the general room temperature. In the second case, the initial temperature inside the isollete box is set as $115^{\circ} \mathrm{F}$ (higher than the maximum safety temperature), and the temperature for the heat actuator is still set as $73^{\circ} \mathrm{F}$. For both of them, the simulation period is set to $0.1 \mathrm{~s}$. Fig. 7(a) and Fig. 7(b) show the simulation results for the two cases respectively, where the blue solid curve and yellow dashed curve represent the trajectories for continuous variables $c$ (for box temperature) and $q$ (for heat temperature) respectively. The simulation results show that, under the control of heat actuator, the temperature inside the isollete box will finally reach a stable state, within the safety range between $97^{\circ} \mathrm{F}$ and $100{ }^{\circ} \mathrm{F}$. The requirement defined in Sec. 3.2 is satisfied.

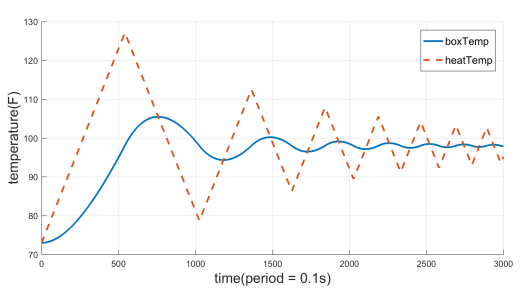

(a) Initial stage: $c=73^{\circ} \mathrm{F}, q=73^{\circ} \mathrm{F}$

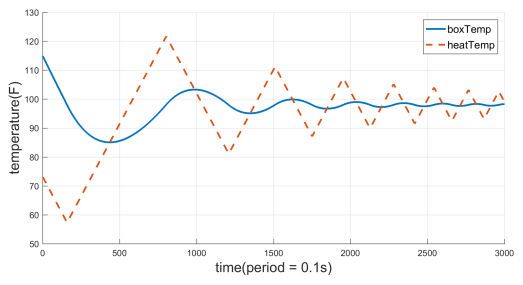

(b) Initial stage: $c=115^{\circ} \mathrm{F}, q=73^{\circ} \mathrm{F}$

Fig. 7: Results of co-simulation from different inital stages 


\section{Related Work}

AADL provides the notion of annex to support extensions to its core language. The key standardized annexes include the Behaviour Annex (BA) which extends AADL with the ability of defining component behaviour via state machines, and BLESS Annex[16], which improves the state transition formalism by introducing assertions for supporting contract-based specifications. The simulation and analysis of AADL models have also been explored a great deal. ADeS was one of the simulation tools with considering the environment in which the system evolves. AADL Inspector produced by Ellidiss company is a powerful software that encompasses various features including schedulability analysis and dynamic simulation. There have also been some work on translation of AADL to other languages for analysis and simulation, e.g. AADL to BIP [9], AADL to Sync [15], AADL to Maude [23] and so on. However, most of them focus on the discrete-time behaviours.

There have been some work on the extension of AADL for hybrid systems. [30] models hybrid systems with AADL based on networks of timed automata, and uses the model checker UPPAAL for property analysis. [25] discusses a sublanguage extension to AADL to describe continuous behaviour, but it has difficulty in modelling complex continuous behaviour expressed with differential equations. In [3], a Hybrid Annex is presented, which is much more expressive in its ability to specify hybrid systems, yet it lacks relevant tools for further simulation and analysis of the hybrid models.

Compared with the above mentioned work, our proposed AADLSim framework coalesces AADL with Simulink for modelling of both discrete and continuous behaviours, and the flexible interaction between them. Moreover, it also provides extensive support for the analysis and simulation of the combined models through translating them into the same target language.

\section{Conclusion and Future work}

In this paper, we propose an unified graphical co-modelling and co-simulation framework for the design of cyber-physical systems. This proposed framework combines AADL and Simulink/Stateflow with which the gap between discrete control and continuous plant can be filled. The combined models are translated into $\mathrm{C}$ code for further analysis by co-simulation. Throughout the paper, we clarify the main concepts of the framework, outline the specific co-simulation flow and the verification process of the combined models. An Isollete system case study is provided to illustrate the framework.

For future work, we will investigate the translation of the combination into HCSP, a formal modelling language encoding hybrid system dynamics by means of an extension of CSP. Formal verification of HCSP is supported by an interactive Hybrid Hoare Logic prover based on Isabelle/HOL. As a consequence, the combined AADL and Simulink/Stateflow models can be verified. To make sure that the generated HCSP model is correct, the consistency between observational behaviours of the models at AADL and Simulink/Stateflow, and HCSP must be guaranteed in a rigorous way. This question, however, is known to be difficult, due to the inherent complexity of hybrid 
systems. To solve this problem, we consider to define the semantics of all the separate models in UTP, to prove their consistency in a unified semantic framework.

\section{References}

1. Simulink User's Guide, 2013. http://www.mathworks.com/help/pdf_doc/ simulink/sl_using.pdf.

2. Stateflow User's Guide, 2013. http://www.mathworks.com/help/pdf_doc/ stateflow/sf_ug.pdf.

3. E. Ahmad, Y. Dong, B. Larson, J. Lü, T. Tang, and N. Zhan. Behavior modeling and verification of movement authority scenario of chinese train control system using aadl. Science China Information Sciences, 58(11):1-20, 2015.

4. E. Ahmad, B. R. Larson, S. C. Barrett, N. Zhan, and Y. Dong. Hybrid annex: An AADL extension for continuous behavior and cyber-physical interaction modeling. In ACM SIGAda Ada Letters, volume 34, pages 29-38, 2014.

5. A. Basu, S. Bensalem, M. Bozga, J. Combaz, M. Jaber, T. Nguyen, and J. Sifakis. Rigorous component-based system design using the BIP framework. IEEE Software, 28(3):41-48, 2011 .

6. A. Benveniste, B. Caillaud, D. Nickovic, R. Passerone, J. Raclet, P. Reinkemeier, A. L. Sangiovanni-Vincentelli, W. Damm, T. A. Henzinger, and K. G. Larsen. Contracts for system design. Foundations and Trends in Electronic Design Automation, 12(2-3):124-400, 2018.

7. M. Chen, X. Han, T. Tang, S. Wang, M. Yang, N. Zhan, H. Zhao, and L. Zou. MARS: A toolchain for modelling, analysis and verification of hybrid systems. In Provably Correct Systems, pages 39-58. Springer, 2017.

8. M. Chen, A. P. Ravn, S. Wang, M. Yang, and N. Zhan. A two-way path between formal and informal design of embedded systems. In UTP'16, volume 10134 of LNCS, pages 65-92. Springer, 2016.

9. M. Chkouri, A. Robert, M. Bozga, and J. Sifakis. Translating AADL into BIP - application to the verification of real-time systems. In MODELS'08, volume 5421 of $L N C S$, pages 5-19. Springer, 2008.

10. J. Delange and P. Feiler. Architecture fault modeling with the AADL error-model annex. In 40th EUROMICRO Conference on Software Engineering and Advanced Applications, pages 361-368. IEEE, 2014.

11. M. D. Ernst, J. H. Perkins, P. J. Guo, S. McCamant, C. Pacheco, M. S. Tschantz, and C. Xiao. The Daikon system for dynamic detection of likely invariants. Science of Computer Programming, 69(1-3):35-45, 2007.

12. P. H. Feiler and D. P. Gluch. Model-Based Engineering with AADL: An Introduction to the SAE Architecture Analysis \& Design Language. Addison-Wesley Professional, 2012.

13. J. He. From CSP to hybrid systems. In A Classical Mind, Essays in Honour of C.A.R. Hoare, pages 171-189. Prentice Hall International (UK) Ltd., 1994.

14. C. A. R. Hoare and J. He. Unifying theories of programming. Prentice Hall, 1998.

15. E. Jahier, N. Halbwachs, P. Raymond, X. Nicollin, and D. Lesens. Virtual execution of AADL models via a translation into synchronous programs. In EMSOFT'07, pages 134143. ACM, 2007.

16. B. R. Larson, P. Chalin, and J. Hatcliff. BLESS: Formal specification and verification of behaviors for embedded systems with software. In NFM'13, volume 7871 of $L N C S$, pages 276-290. Springer, 2013.

17. D. L. Lempia and S. P. Miller. Requirements engineering management handbook. National Technical Information Service (NTIS), 2009. 
18. J. Liu, J. Lv, Z. Quan, N. Zhan, H. Zhao, C. Zhou, and L. Zou. A calculus for hybrid CSP. In APLAS'10, volume 6461 of $L N C S$, pages 1-15. Springer, 2010.

19. J. Liu, N. Zhan, and H. Zhao. Computing semi-algebraic invariants for polynomial dynamical systems. In Proceedings of the ninth ACM international conference on Embedded software, pages 97-106. ACM, 2011.

20. S. Lunel. Parallelism and modular proof in differential dynamic logic. (Parallélisme et preuve modulaire en logique dynamique différentielle). $\mathrm{PhD}$ thesis, University of Rennes 1, France, 2019.

21. S. Lunel, B. Boyer, and J. Talpin. Compositional proofs in differential dynamic logic dL. In ACSD'17, pages 19-28, 2017.

22. B. Meyer. Object-oriented Software Construction (2Nd Ed.). Prentice-Hall, Inc., 1997.

23. P. Ölveczky, A. Boronat, and J. Meseguer. Formal semantics and analysis of behavioral AADL models in real-time Maude. In Formal Techniques for Distributed Systems, volume 6117 of $L N C S$, pages 47-62. Springer, 2010.

24. A. Platzer. Logical Foundations of Cyber-Physical Systems. Springer, 2018.

25. Y. Qian, J. Liu, and X. Chen. Hybrid AADL: A sublanguage extension to AADL. In Internetware '13. ACM, 2013.

26. SAE International Standards. Aarchitecture analysis \& design language (AADL), Revision B. 2012.

27. S. Wang, N. Zhan, and D. Guelev. An assume/guarantee based compositional calculus for hybrid CSP. In TAMC'12, volume 7287 of $L N C S$, pages 72-83. Springer, 2012.

28. S. Wang, N. Zhan, and L. Zou. An improved HHL prover: an interactive theorem prover for hybrid systems. In ICFEM'15, volume 9407 of LNCS, pages 382-399. Springer, 2015.

29. N. Zhan, S. Wang, and H. Zhao. Formal Verification of Simulink/Stateflow Diagrams. Springer, 2017.

30. Y. Zhang, Y. Dong, F. Zhang, and Y. Zhang. Research on modeling and analysis of CPS. In International Conference on Autonomic and Trusted Computing, pages 92-105. Springer, 2011.

31. C. Zhou, J. Wang, and A. P. Ravn. A formal description of hybrid systems. In Hybrid systems, volume 1066 of $L N C S$, pages 511-530, 1996.

32. L. Zou, J. Lv, S. Wang, N. Zhan, T. Tang, L. Yuan, and Y. Liu. Verifying chinese train control system under a combined scenario by theorem proving. In VSTTE'13, volume 8164 of $L N C S$, pages 262-280, 2013.

33. L. Zou, N. Zhan, S. Wang, and M. Fränzle. Formal verification of Simulink/Stateflow diagrams. In ATVA'15, volume 9364 of LNCS, pages 464-481. Springer, 2015.

34. L. Zou, N. Zhan, S. Wang, M. Fränzle, and S. Qin. Verifying Simulink diagrams via a hybrid Hoare logic prover. In EMSOFT'13, pages 1-9. IEEE, 2013. 